\title{
Phosphorylation and partial sequence of pregnant sheep myometrium myosin light chain kinase
}

\author{
Mary D. Pato, Ewa Kerc and Stephen J. Lye ${ }^{1}$ \\ Department of Biochemistry, University of Saskatchewan, Saskatoon, Saskatchewan, S7N OWO; 'Division of Perinatology, \\ Samuel Lunenfeld Research Institute, Mount Sinai Hospital, Toronto, Ontario, M5G 1X5 Canada
}

\begin{abstract}
The function of the uterine smooth muscle in gestation and parturition is affected by a variety of hormones and biomolecules, some of which alter the intracellular levels of $\mathrm{cAMP}$ and $\mathrm{Ca}^{2+}$. Since the activity of smooth muscle MLCK has been shown to be modulated by phosphorylation, the effect of this modification of pregnant sheep myometrium (psm) MLCK by the catalytic subunit of cAMP-dependent protein kinase (PKA) and protein kinase C (PKC) was studied. In contrast to other smooth muscle MLCK reported, PKA incorporates 2.0-2.2 moles phosphate into a mole of psm MLCK both in the presence and absence of $\mathrm{Ca}^{2+}$-calmodulin. Modification of serine residues inhibited the activity of the enzyme. PKC also incorporated 2.0-2.1 moles of phosphate per mole psmMLCK under both conditions but had no effect on the MLCK activity. Sequential phosphorylation by PKC and PKA incorporated 3.8-4.1 moles phosphate suggesting that the amino acid residues modified by the two kinases are different. Phosphoamino acid analysis of the MLCK revealed that PKC phosphorylated serine and threonine residues. The double reciprocal plots of the enzyme activity and calmodulin concentrations showed that the $\mathrm{V}_{\max }$ of the reaction is not altered by phosphorylation by PKA but the calmodulin concentration require for half-maximal activation is increased about 4-fold. Only 10 out of 17 monoclonal antibodies to various regions of the turkey gizzard MLCK cross-reacted with psmMLCK suggesting structural differences between these enzymes. Comparison of the deduced amino acid sequence of the cDNA encoding the C-terminal half of the psmMLCK molecule showed that while cgMLCK and psmMLCK are highly homologous, a number of nonconservative substitutions are present, particularly near the PKA phosphorylation site B $\left(\mathrm{S}^{828}\right)$. (Mol Cell Biochem 149/150: 59-69, 1995)
\end{abstract}

Key words: myometrium, myosin light chain kinase, calmodulin, phosphorylation

Abbreviations: MLCK - myosin light chain kinase; psm - pregnant sheep myometrium; tg - turkey gizzard; cg - chicken gizzard; ru - rabbit uterine; bs - bovine stomach; PKA - catalytic subunit of cAMP-dependent protein kinase; PKC - $\mathrm{Ca}^{2+}$ and phospholipid dependent protein kinase; CaM Kinase II - multifunctional $\mathrm{Ca}^{2+}$ and calmodulin dependent protein kinase

\section{Introduction}

Phosphorylation of the $20,000 \mathrm{Da}$ light chains of myosin by a calcium-calmodulin dependent enzyme, Myosin Light Chain Kinase (MLCK) is essential for the initiation and regulation of contractile activity in smooth muscles [1]. MLCK has been purified from skeletal, cardiac, and smooth muscles, and non-muscle cells [2]. A common property of these enzymes is their requirement for $\mathrm{Ca}^{2+}$ and calmodulin for activation. They have a narrow substrate specificity for the 20
$\mathrm{kD}$ light chain of myosin, the only protein observed to be phosphorylated at a rapid rate. The structural properties of MLCK from skeletal muscle and avian gizzards have been extensively characterized. The complete amino acid sequence of the rabbit skeletal muscle MLCK has been determined [3, 4] and its functional domains delineated [5]. The cDNAs encoding MLCK from rat skeletal muscle [6], chicken gizzard [7], chicken embryo fibroblast (8), rabbit uterine smooth muscle [9] and bovine stomach [10] have been isolated, sequenced and characterized. 
Purification and characterization of MLCK from pregnant sheep myometrium revealed that like other MLCK reported, it requires calmodulin for activation but its molecular weight $(160,000)$ is higher than most MLCK [11]. Structural difference between the avian and pregnant sheep myometrium (psm) MLCK is suggested by the observation that some polyclonal antibodies prepared against psmMLCK did not cross-react with turkey gizzard $(\mathrm{tg})$ and chicken gizzard $(\mathrm{cg})$ MLCK. The epitopes of these antibodies are mainly localized on a $28 \mathrm{kD}$ peptide at the $\mathrm{N}$-terminal region as suggested by immunoblot analysis of the tryptic digests of psmMLCK. To identify other differences between psmMLCK and the avian enzymes, the phosphorylation of these enzymes by the catalytic subunit of the cyclic AMP dependent protein kinase (PKA) and the $\mathrm{Ca}^{2+}$ and phospholipid dependent protein kinase, Protein Kinase C (PKC) were compared.

This paper reports that phosphorylation of psmMLCK by PKA, both in the presence and absence of $\mathrm{Ca}^{2+}$-calmodulin, incorporated two moles of phosphate and decreased the activity of the enzyme. On the other hand, phosphorylation of psmMLCK by PKC in the presence and absence of $\mathrm{Ca}^{2+}$ calmodulin did not alter the activity of the enzyme. To understand the biochemical basis for these observations, the cDNA encoding the C-terminal region of psmMLCK which contains the catalytic domain, calmodulin binding site, pseudosubstrate region and PKA phosphorylation sites was cloned, sequenced and analyzed.

\section{Materials and methods}

\section{Preparation of proteins}

Myosin light chain kinase was prepared from fresh turkey gizzard according to the procedure described by Adelstein and Klee [12]. The $40-60 \%\left(\mathrm{NH}_{4}\right)_{2} \mathrm{SO}_{4}$ fraction of the extract was subjected to gel filtration on Sephacryl S-300, ionexchange on DEAE-Sephacel and affinity chromatography on Calmodulin-Sepharose. A modified procedure was used for the purification of MLCK from myometrium of pregnant sheep killed in the late gestation period (135-145 days) [12]. Frozen tissues was used in the latter procedure and proteolytic inhibitors were included in all buffers used in the preparation. Smooth Muscle Phosphatase-I was purified from turkey gizzard [13] while calmodulin was prepared from bovine brain [14]. The $20 \mathrm{kD}$ light chains of myosin were isolated from turkey gizzards [15] and separated from contaminating calmodulin by affinity chromatography on tg MLCKSepharose in the presence of $\mathrm{Ca}^{2+}$. PKA was prepared from rat skeletal muscle as described in [16]. The resins used for the purification of the proteins were purchased from Pharmacia Fine Chemicals. The concentration of the proteins used in this study were determined by the Bradford method [17].

\section{Phosphorylation of MLCK}

Phosphorylation of MLCK by PKA in the presence of $\mathrm{Ca}^{2+}$ calmodulin was carried out in a reaction mixture $(200-300$ $\mu \mathrm{l})$ containing $0.6-1.2 \mu \mathrm{M}$ MLCK in $50 \mathrm{mM}$ Tris. $\mathrm{HCl}, 10$ $\mathrm{mM} \mathrm{Mg}^{2+}, 0.1 \mathrm{mM}\left[{ }^{32} \mathrm{P}\right] \mathrm{ATP}, 0.2 \mathrm{mM} \mathrm{Ca}^{2+}, 3 \mu \mathrm{M}$ calmodulin incubated at room temperature. Following addition of PKA, aliquots of the reaction mixture were taken at 2, 10, 30,60 min for analysis on SDS-polyacrylamide gel and determination of phosphate incorporation. At $30 \mathrm{~min}$, half of the reaction mixture was made to $1 \mathrm{mM}$ EGTA and aliquots of the resulting reaction mixture were taken for analysis at 32,40 and $60 \mathrm{~min}$. The unphosphorylated enzyme (control) was also subjected to the same treatment except for the omission of PKA in the reaction mixture. Phosphorylation of MLCK in the absence of $\mathrm{Ca}^{2+}$-calmodulin was carried out, under 3 different conditions, in the same reaction mixture described above but (a) containing $1 \mathrm{mM}$ EGTA, (b) without the addition of $\mathrm{Ca}^{2+}$, and (c) without the addition of $\mathrm{Ca}^{2+}$ and calmodulin. Samples for SDS-polyacrylamide gel electrophoresis were mixed with equal volume of $0.1 \mathrm{M}$ Tris. $\mathrm{HCl}$ ( $\mathrm{pH} 6.8$ ), $2 \%$ SDS, $20 \%$ glycerol, bromophenol blue, boiled immediately, and subsequently, analyzed on a $12.5 \%$ SDS-polyacrylamide gel according to the procedure of Laemmli [18]. The samples for the determination of phosphate incorporation were spotted on a strip $(2 \times 2 \mathrm{~cm})$ of phosphocellulose paper (Whatman P81 ion exchange chromatography paper) [19]. The paper strips were immersed immediately in $75 \mathrm{mM}$ $\mathrm{H}_{3} \mathrm{PO}_{4}$, washed with the same solution three times and counted in a scintillation counter. Phosphorylation of MLCK by PKC (gift of Dr. James R. Sellers, NIH) was carried out in reaction mixture containing $25 \mathrm{mM} \mathrm{Tris.} \mathrm{HCl}(\mathrm{pH} 7.5), 0.1$ $\mathrm{mM}\left[{ }^{32} \mathrm{p}\right]$ ATP, $0.2 \mathrm{mM} \mathrm{Ca}^{2+}, 10 \mu \mathrm{g} / \mathrm{ml}$ phosphatidylserine and $1 \mu \mathrm{g} / \mathrm{ml}$ diolein, as described by Nishikawa et al. [20]. Aliquots of reaction mixture were taken for determination of kinase activity, phosphate incorporation, SDS polyacrylamide gel electrophoresis and phosphoamino acid analysis.

\section{Peptide mapping}

An aliquot of the phosphorylation reaction mixture was made to $6 \mathrm{M}$ guanidine hydrochloride to terminate the phosphorylation reaction and denature the proteins. It was incubated for $60 \mathrm{~min}$ at $37^{\circ} \mathrm{C}$ and dialyzed against $0.5 \mathrm{M} \mathrm{KCl}, 0.2 \mathrm{M}$ $\mathrm{NH}_{4} \mathrm{HCO}_{3}(\mathrm{pH} 8.0$ ), 1 mM EGTA, $5 \mathrm{mM}$ dithiothreitol overnight with several changes [21]. Tryptic digestion of the enzyme was performed by the addition of L-tosylamido2-phenylethyl chloromethyl ketone-treated trypsin at a 
trypsin to substrate ratio of $1: 50$ and incubated at $37^{\circ} \mathrm{C}$ for $16 \mathrm{~h}$. The digest was lyophilyzed, dissolved in $2 \% \mathrm{NH}_{4} \mathrm{OH}$ and electrophoresced on a thin layer cellulose sheet (Eastman, $20 \times 20 \mathrm{~cm})$ at $4^{\circ} \mathrm{C}, 1000 \mathrm{~V}$ for $105 \mathrm{~min}$ in acetic acid: formic acid: $\mathrm{H}_{2} \mathrm{O}(15: 5: 80) \mathrm{pH} 1.9$ [20]. The cellulose sheet was dried and autoradiographed.

\section{Identification of the phosphoamino acid}

The identification of the phosphoamino acid was carried out according to the procedure of de Lanerolle and Nishikawa [22]. Aliquots of the phosphorylated psmMLCK were lyophilized and hydrolyzed with $6 \mathrm{M} \mathrm{HCl}$ at $110^{\circ} \mathrm{C}$ for $4 \mathrm{~h}$. The $\mathrm{HCl}$ hydrolysates were lyophilized, dissolved in water and analyzed by high voltage thin layer chromatography on cellulose sheet at $\mathrm{pH} 3.5$ in pyridine:acetic acid: $\mathrm{H}_{2} \mathrm{O}(1: 10: 189)$ for $45 \mathrm{~min}$ at 1000 volts. Following chromatography, the cellulose sheet was dried and autoradiographed. The phosphoamino acids were identified by comparison with standards, phosphoserine and phosphothreonine, which were visualized with ninhydrin.

\section{MLCK activity assay}

The effect of phosphorylation on the activity of the MLCK was determined by assaying aliquots taken after phosphorylation by PKA or PKC in the presence and absence of $\mathrm{Ca}^{2+}$ calmodulin as described above but using nonradioactive ATP. The MLCK activity was determined in a reaction mixture (50 $\mu \mathrm{l})$ containing $50 \mathrm{mM}$ Tris. $\mathrm{HCl}(\mathrm{pH} 7.4), 10 \mathrm{mM} \mathrm{MgCl}_{2}, 0.2$ $\mathrm{mM} \mathrm{CaCl}, 0.1 \mathrm{mM}\left[{ }^{32} \mathrm{p}\right] \mathrm{ATP}, 50-100 \mu \mathrm{M} 20 \mathrm{kD}$ myosin light chains, $1 \mathrm{mM}$ dithiothreitol and varying concentrations of calmodulin at room temperature. The reaction was initiated by the addition of MLCK. The reaction time was determined from the linear portion of the activity curve of the unphosphorylated MLCK with respect to time in the presence of 100 $\mathrm{nM}$ calmodulin. Aliquots of the reaction mixture were applied to strips of phosphocellulose paper, washed with $\mathrm{H}_{3} \mathrm{PO}_{4}$ and counted in a scintillation counter.

\section{Immunoassay}

Immunodot blot analysis was performed by spotting equal volumes of purified psmMLCK to pieces of nitrocellulose paper. The nitrocelulose blots were blocked with 3\% gelatin for $1 \mathrm{~h}$, incubated separately with 17 different monoclonal anti tgMLCK antibodies (gift of Dr. M. Ikebe, Case Western University) for $4 \mathrm{~h}$ and then incubated with immunoglobulin conjugated with alkaline phosphatase for $1 \mathrm{~h}$. After washing the nitrocellulose blots with $20 \mathrm{mM}$ Tris. $\mathrm{HCl}(\mathrm{pH} 7.5)$,
$500 \mathrm{mM} \mathrm{NaCl}, 0.05 \%$ Tween, the secondary antibodies were detected by addition of the substrates of alkaline phosphatase, 5-bromo-4-chloro-3-indoyl phosphate toluidine salt and pnitro blue tetrazolium chloride.

\section{Construction of sheep myometrial cDNA library}

Total RNA was obtained from myometrial tissue collected from a pregnant sheep at day 127 of gestation. The myometrial tissue was snap frozen in liquid nitrogen and total RNA was extracted from the tissue according to a modification of the method of Chirgwin et al. [23] using a cesium trifluoroacetate (Pharmacia) density gradient.

PolyA $^{+}$RNA was isolated from the sheep myometrial total RNA using Stratagene Poly A Quik Kit. The quality of this messenger RNA was assessed by Northern analysis using PCR product (see below) as probe. The mRNA was used to generate a cDNA library using the UniZap cDNA Library kit (Stratagene). The cDNA library was packaged using the Lamda Zap packaging kit (Stratagene).

\section{Preparation of oligonucleotide probes by PCR}

Degenerate oligonucleotides were synthesized based on sequences of the catalytic domain (amino acid residues 639645 ) and calmodulin binding domain (amino acid residues 800-805) of cgMLCK [7] which show high degree of sequence similarity between chicken gizzard and rat skeletal MLCK. These sequences are about $500 \mathrm{bp}$ apart in the cgMLCK. A Sal 1 site was added to the 5'end of the catalytic domain oligonucleotide, 5'GGGTCGACc/tTc/gAAGCCAGAa/gAA3', and a Cla 1 site was added to the 5'end of the calmodulin binding domain oligonucleotide, 5'GGATCGATGCAt/at/gGc/ac/ag/tg/tTTTTCTt/gCC3'.

Total myometrial RNA was primed with an oligo $\mathrm{dT}$ primer (Pharmacia) and transcribed using Moloney Murine Leukemia Virus reverse transcriptase (Boehringer Mannheim) at $37^{\circ} \mathrm{C}$ for $30 \mathrm{~min}$. Polymerase Chain Reaction was performed using the above synthetic oligonucleotide primers and Taq polymerase (Promega). The Perkin Elmer Cetus thermal cycler was programed to cycle 30 times: denaturing at $95^{\circ} \mathrm{C}$ for $60 \mathrm{sec}$, annealing at $59^{\circ} \mathrm{C}$ for $30 \mathrm{sec}$ and elongating at $72^{\circ} \mathrm{C}$ for $2 \mathrm{~min}$. The PCR products were cut using $\mathrm{Cla} 1$ and Sal 1 (Boehringer Mannheim) and the digests were electrophoresced on a TAE low melting point agarose minigel. A DNA band around the expected size of $500 \mathrm{bp}$ was cut from the gel and extracted using Geneclean reagents (BioRad). The PCR fragments were ligated into pSP72 and the recombinant DNA were transformed in DH5 $\alpha$ cells. Ampicillin resistant colonies were amplified for plasmid isolation and purification using standard protocol [24]. Clones were verified by 
hybridizing a random primed cgMLCK cDNA (gift of Dr. A. R. Means, Baylor College of Medicine) to a blot of a minigel containing the $\mathrm{Cla} 1 / \mathrm{Sal} 1$ digested plasmids. Clones that hybridized to the cgMLCK cDNA probe were sequenced using the Sequenase kit (United States Biochemical) and were found to have a very high degree of similarity to that of the cgMLCK sequence.

\section{Screening of cDNA library and sequencing of smMLCK clone}

The original cDNA library was amplified once. Then $2 \times 10^{5}$ phage were plated, and lifted and fixed onto nylon membranes (Amersham). Primary screening of the membranes with the PCR products revealed 4 positive plaques. Two of these plaques were rescreened and isolated as Lambda DNA in pBluescript (Stratagene). The pBluescript clones were characterized by sequencing their ends, mapping their restriction sites and determining their ability to hybridize to the PCR products by Southern Blot analysis. The complete sequence of the longer clone $2 \mathrm{~B}(1.4 \mathrm{~kb})$ was determined using the M13 sequencing vectors $\mathrm{mp} 18$ and $\mathrm{mp} 19$, and the extension reagents provided with the Sequenase kit (United States Biochemicals).

\section{Results}

Figure 1 shows a comparison of the time course of phosphorylation of tgMLCK which was used as control, and psmMLCK by PKA in the presence and absence of $\mathrm{Ca}^{2+}$ calmodulin. As previously described by Conti and Adelstein [21], one mole of phosphate is incorporated into tgMLCK in the presence of $\mathrm{Ca}^{2+}$-calmodulin and 2 moles phosphate in the absence of $\mathrm{Ca}^{2+}$-calmodulin (Fig. 1A). Similarly, 2.2 moles phosphate were incorporated into a mole of psmMLCK in the absence of $\mathrm{Ca}^{2+}$-calmodulin but in contrast to tgMLCK, 2.0 moles of phosphate were also incorporated in the presence of $\mathrm{Ca}^{2+}$-calmodulin (Fig. 1B) suggesting that the two sites of phosphorylation in psmMLCK are accessible to PKA under both conditions. Addition of EGTA to the reaction mixture containing $\mathrm{Ca}^{2+}$-calmodulin after $30 \mathrm{~min}$ of incubation to dissociate the complex, resulted only in a slight increase of phosphorylation of psmMLCK but incorporation of another mole of phosphate into tgMLCK. The phosphorylation in the absence of $\mathrm{Ca}^{2+}$-calmodulin was carried out in three different conditions: in a reaction mixture containing $\mathrm{Ca}^{2+}$-calmodulin and $1 \mathrm{mM}$ free EGTA; in the presence of calmodulin but no $\mathrm{Ca}^{2+}$; and in the absence of both $\mathrm{Ca} 2+$ and calmodulin. In all cases, the time course of phosphorylation observed were similar to that shown in Fig. 1B.

The aliquots of the phosphorylation reaction mixtures in
A.

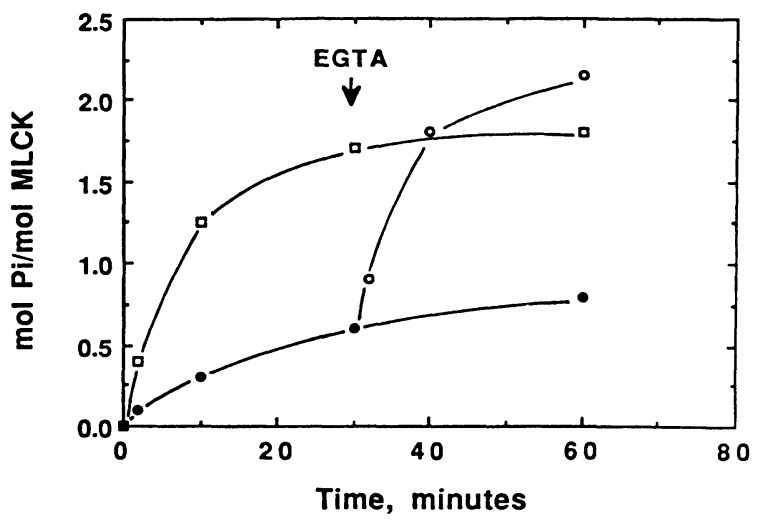

B.

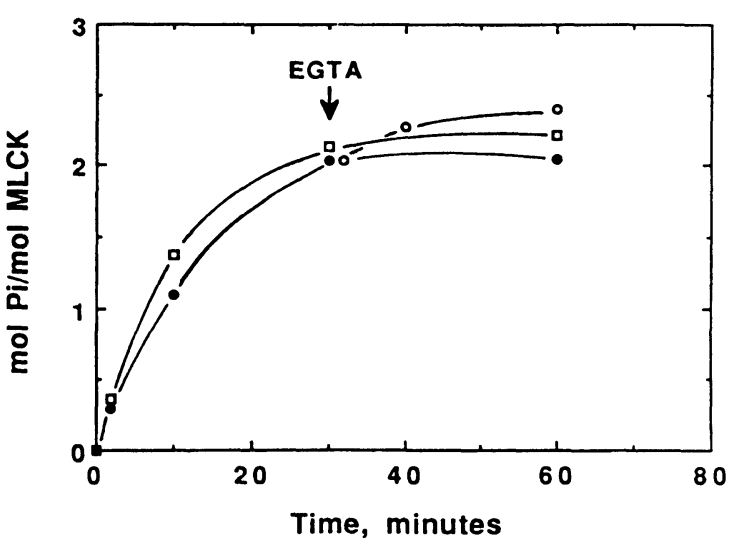

Fig. 1. Time course of phosphorylation of MLCK from turkey gizzards (A) and pregnant sheep myometrium (B) by PKA in the absence ( $\square$ ) and presence $(\bullet)$ of bound $\mathrm{Ca}^{2+}$-calmodulin as described in the "Materials and methods'. After $30 \mathrm{~min}$ of phosphorylation, half of the latter reaction mixture containing $\mathrm{Ca}^{2+}$-calmodulin was made to $1 \mathrm{mM}$ EGTA (O). Aliquots of the reaction mixtures were taken at the times indicated for determination of phosphate incorporation.

Figs $1 \mathrm{~A}$ and $\mathrm{B}$ taken at 0,30 and 60 min were analyzed on SDS-polyacrylamide gels. The autoradiographs of these gels show that all the radioactivity were confined to the MLCK band and confirmed the lack of significant difference in the extent of phosphorylation of psmMLCK in the presence and absence of $\mathrm{Ca}^{2+}$-calmodulin. As expected, the phosphate incorporation into tgMLCK in the absence of $\mathrm{Ca}^{2+}$-calmodulin is about twice that in the presence of $\mathrm{Ca}^{2+}$-calmodulin.

To eliminate the possibility that the purified psmMLCK is already partially phosphorylated, psmMLCK was incubated with Smooth Muscle Phosphatase-I [13] from turkey gizzards for $60 \mathrm{~min}$ at $30^{\circ} \mathrm{C}$. This enzyme has been shown previously to dephoshorylate MLCK which has been phosphorylated by PKA. The phosphatase was then inhibited by the addition of okadaic acid, a potent phosphatase inhibitor [25] which did not affect the activities of the MLCK and PKA. Phosphorylation of the phosphatase-treated-psmMLCK by PKA in the presence and absence of $\mathrm{Ca}^{2+}$-calmodulin gave the same re- 
sults as that described in Fig. 1B, proving that the discrepancy observed between the psmMLCK and tgMLCK is not due to a partially phosphorylated psmMLCK (data not shown).

Comparison of the tryptic peptide maps of the psmMLCK phosphorylated in the presence and absence of $\mathrm{Ca}^{2+}$ calmodulin with that of the diphosphorylated tg-MLCK revealed that the phosphopeptides of psmMLCK have the same mobility as the tryptic phosphopeptides of tgMLCK phosphorylated in the absence of $\mathrm{Ca}^{2+}$-calmodulin (Fig. 2) suggesting that the sites of phosphorylation of tg- and psmMLCK by PKA are the same. Analysis of the $\mathrm{HCl}$ hydrolysate of the phosphorylated psmMLCK by high voltage thin layer electrophoresis showed that the amino acids modified by PKA in the presence and absence of $\mathrm{Ca}^{2+}$ - a

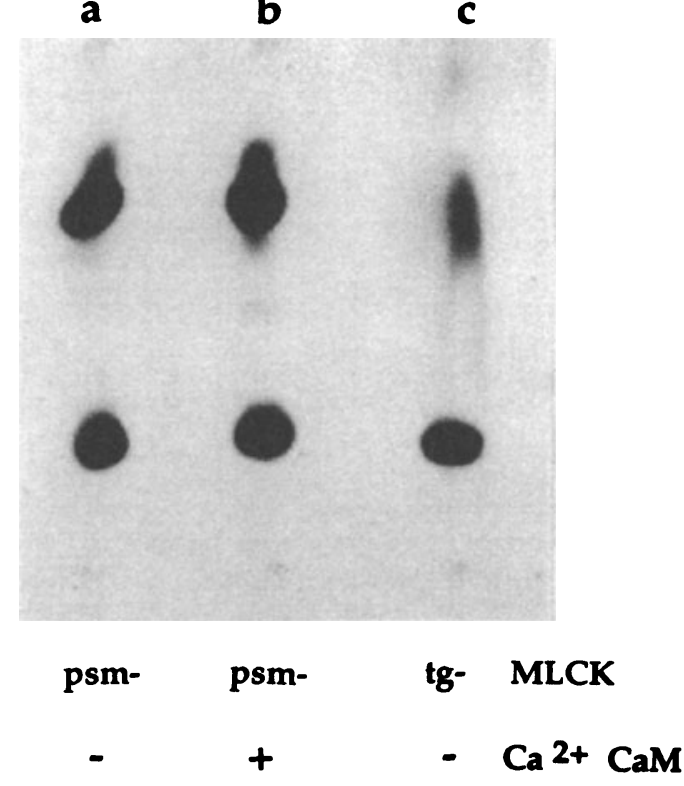

Fig. 2. Autoradiograph of ${ }^{32} \mathrm{P}$-labeled tryptic peptides of psmMLCK after thin layer cellulose electrophoresis. Aliquots of the reaction mixtures in Fig. 1 were taken after 60 min incubation, lyophilized and digested with trypsin as described in the 'Materials and methods'. The tryptic peptides of psmMLCK (lanes a and b) and tgMLCK (lane c) phosphorylated by PKA in the absence (lanes a and c) and presence (lane b) of $\mathrm{Ca}^{2+}$. calmodulin were subjected to thin layer electrophoresis.

calmodulin are serine residues (data not shown).

Nishikawa et al. [20] reported that phosphorylation of tgMLCK by PKC altered the activity of the enzyme while Ikebe et al. [26] did not observe any effect of phosphorylation. Phosphorylation of psmMLCK with PKC in the presence and absence of $\mathrm{Ca}^{2+}$-calmodulin resulted in the incorporation of 2.0-2.1 moles phosphate into one mole of enzyme under both conditions (Fig. 3). Addition of PKA to psmMLCK phosphorylated with PKC resulted in further phosphate incorporation to a total of about $4 \mathrm{~mol}$ phosphate $/ \mathrm{mol}$

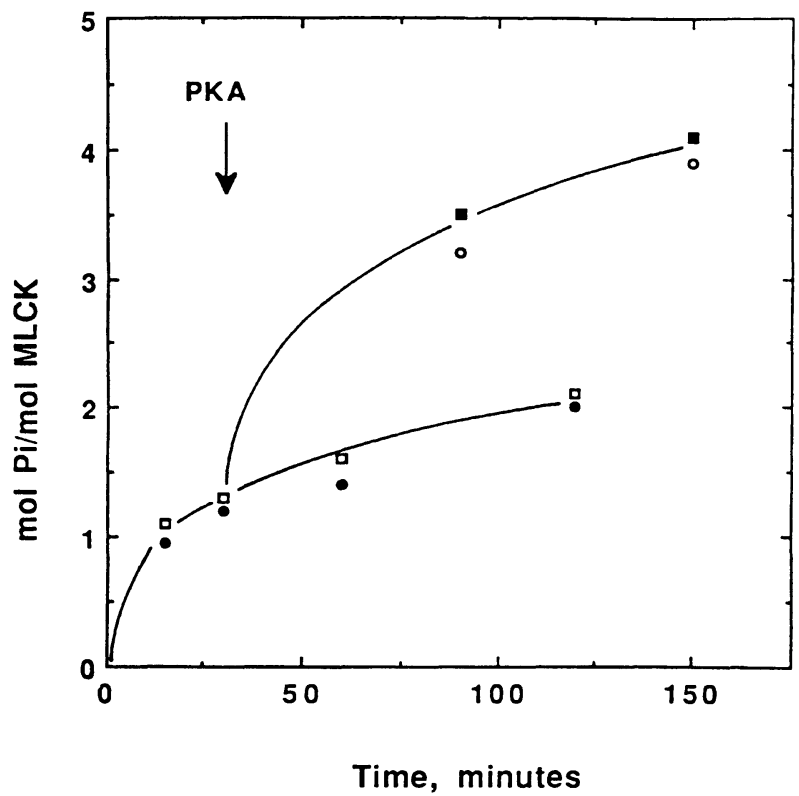

Fig. 3. Time course of phosphorylation of psmMLCK by PKC and PKA. Phosphorylation of psmMLCK by PKC was carried out in the presence $(\square)$ and absence $(\bullet)$ of $\mathrm{Ca}^{2+}$-calmodulin. After $30 \mathrm{~min}$ of incubation, PKA was added to half of the reaction mixtures $(\square, 0)$. Aliquots were taken for determination of phosphate incorporation.

enzyme suggesting that the sites of phosphorylation by the PKA and PKC are different. This conclusion is supported by the results of the phosphoamino acid analysis showing that one of the amino acids modified by PKC is a threonine residue (Fig. 4).

To determine the effect of phosphorylation on the kinetic properties of psmMLCK, the activities of the phosphorylated and unphosphorylated psmMLCK were assayed at varying calmodulin concentrations. Figure 5A shows that the activity curves of the enzyme phosphorylated by PKA in the presence and absence of $\mathrm{Ca}^{2+}$-calmodulin are the same, and that the phosphorylated enzyme requires higher calmodulin concentration for activation than the unphosphorylated species. The double reciprocal plots of the activity of MLCK and concentration of calmodulin showed that phosphorylation of psmMLCK by PKA does not have an effect on the $\mathrm{V}_{\text {max }}$ of the reaction but increases the $\mathrm{K}_{\text {CAM }}$ 3-fold from 3-9.2 nM (data not shown). In contrast, phosphorylation by PKC did not affect the calmodulin activation dose response curve (Fig. 5B), and the $\mathrm{K}_{\mathrm{CAM}}$ and $\mathrm{V}_{\max }$ of the reaction (Fig. 6). Figure 6 also shows that the kinetic properties of psmMLCK phosphorylated by PKA is not affected by prephosphorylation by PKC.

The disimilarity of the effect of phosphorylation of psmMLCK and tgMLCK by PKA and PKC may be due to differences in the structural properties of these enzymes. To verify this possibility, the cross-reactivity of psmMLCK with 17 monoclonal antibodies which have been demonstrated to 


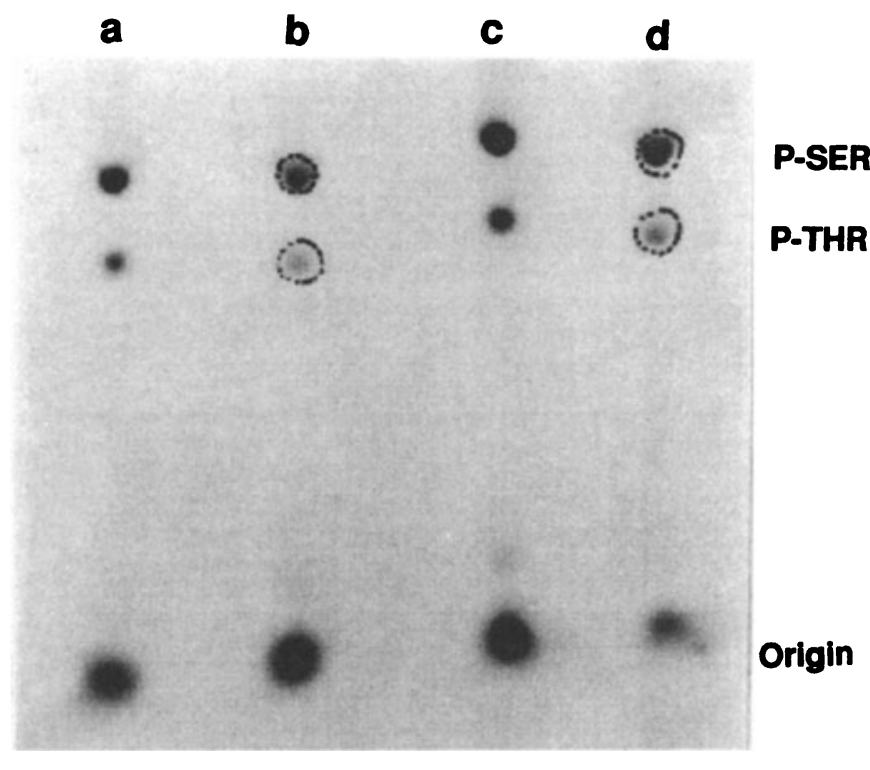

Fig. 4. Identification of the amino acids phosphorylated by PKC. psmMLCK samples which were phosphorylated by PKC (Fig. 3) in the presence (lanes $\mathrm{a}$ and $\mathrm{b}$ ) and absence (lanes $\mathrm{c}$ and d) of $\mathrm{Ca}^{2+}$-calmodulin were digested with $\mathrm{HCl}$. The hyrolysates were electrophoresed on a thin layer cellulose sheet at $\mathrm{pH} 3.5$ at $1000 \mathrm{~V}$ for $45 \mathrm{~min}$. Standard amino acids, phosphoserine and phosphothreonine were added to the samples applied to lanes $b$ and $c$ and were visualized by ninhydrin (broken circle). The ${ }^{32} \mathrm{p}$ labelled amino acids were detected by autoradiography.

cross-react with different regions of tgMLCK molecule by Araki and Ikebe [27] was studied. Immunodot blot analysis showed that only 10 of these antibodies cross-reacted strongly with psmMLCK (data not shown). The epitopes of 5 of these antibodies, $\mathrm{mAb}$ LKH 2, 4, 8, 19, and 20, may be at the Nterminal region or at the calmodulin binding site while those of LKH 7, 10 and 14 are on the $64 \mathrm{kD}$ tryptic peptide of tgMLCK. LKH $18 \mathrm{mAb}$ which cross-reacts with the calmodulin binding region and LKH 16 which cross-reacts with all the tryptic peptides of tgMLCK tested also bound to psmMLCK. Very weak interaction, if any, was observed with the other antibodies with epitopes at the $\mathrm{N}$-terminal region or calmodulin binding site (LKH 6, 17, 21 and 22) and on the $64 \mathrm{kD}$ tryptic peptide (LKH 15). Interestingly, $2 \mathrm{mAb}$ with epitopes on the $23 \mathrm{kD}$ tryptic phosphopeptide (LKH 1 and 3) did not cross-react with psmMLCK. These results provide direct evidence for the presence of structural differences between these enzymes.

To compare further the structural properties of the avian and psmMLCK, the cDNA encoding the $\mathrm{C}$-terminal region of psmMLCK was cloned and sequenced. The nucleotide and amino acid sequences of this clone are shown in Figs 7A and $\mathrm{B}$, respectively, and are compared to equivalent regions of cgMLCK [7] and ruMLCK [9]. The psmMLCK cDNA encodes the region from the amino acid residue 553 of cgMLCK or 711 of ruMLCK to the C-terminus. This region encompasses part of the catalytic domain ( cgMLCK 526-762),
A.

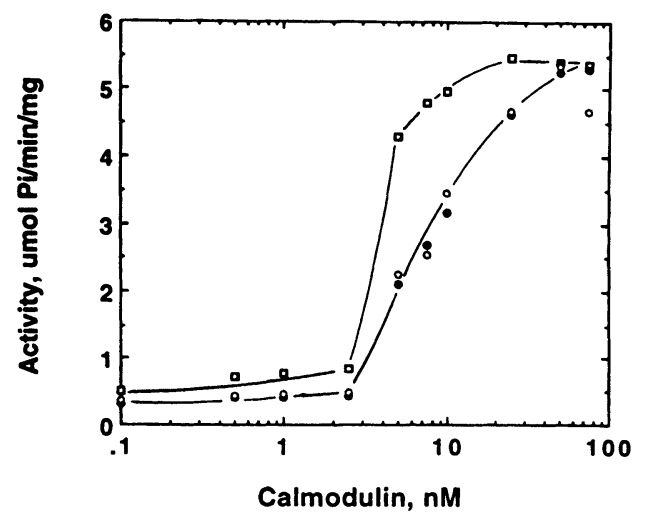

B.

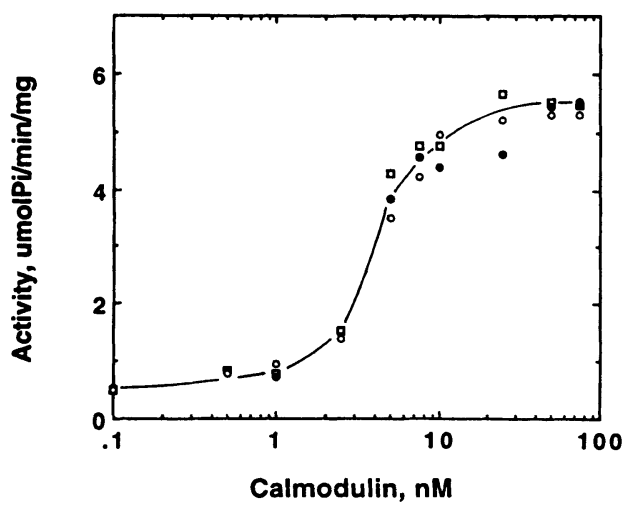

Fig. 5. Activation by calmodulin of unphosphorylated psmMLCK ( $\square$ ), psmMLCK phosphorylated in the presence $(\bullet)$ and absence $(O)$ of $\mathrm{Ca}^{2+}-$ calmodulin by PKA (A) and PKC (B). Following incubation of psmMLCK with PKA for $60 \mathrm{~min}$ as described in Fig. 1B and with PKC for $2 \mathrm{~h}$ as described in Fig. 3, the MLCK activity was determined at various calmodulin concentration.

psuedosubstrate domain (cgMLCK 787-807) as well as the PKA phosphorylation site A $\left(\mathrm{Ser}^{815}\right)$ and site $\mathrm{B}\left(\mathrm{Ser}^{828}\right)$.

The homology of the nucleotide sequence of the psmMLCK to that of ruMLCK is $91.2 \%$, and $78.9 \%$ to that of cgMLCK. Of the 438 amino acids encoded by the psmMLCK cDNA, only 12 residues $(2.7 \%)$ differ from those of ruMLCK and 33 residues $(7.5 \%)$ from those of cgMLCK. Interestingly, 5 of the 12 amino acids differences between ru- and psmMLCK are unchanged in the cgMLCK. Of the 33 amino acids differences between the ru- and cgMLCK, 8 amino acids are unchanged in psmMLCK. Many of these amino acid substitutions are nonconservative. More recently, the sequence of the cDNA of the $155 \mathrm{kDa}$ bovine stomach (bs) MLCK was reported [10]. Comparison of the sequence of the psmMLCK with the same region of the bsMLCK (F740 to the C-terminus) revealed only 4 amino acid differences. A glycine residue at position 1142 of psmMLCK and 1170 of bsMLCK is not present in either ru- and cgMLCK. Furthermore, there are two sets of 3 amino acids in the cgMLCK sequence (at 1134 


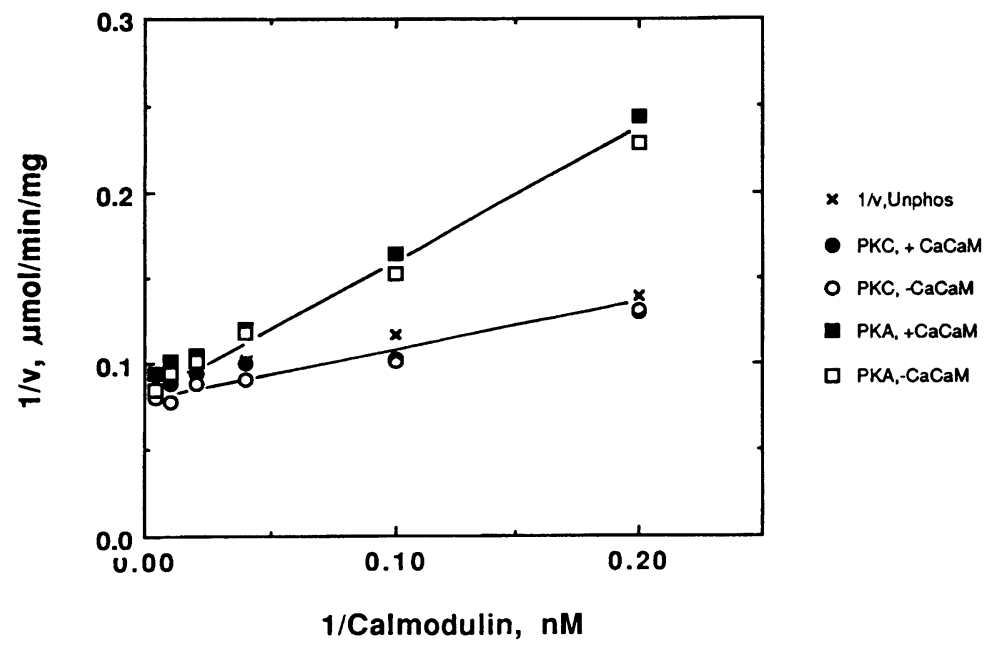

Fig. 6. Double reciprocal plots of the activities of unphosphorylated (x) and phosphorylated psmMLCK at varying calmodulin concentrations. The activities of psmMLCK phosphorylated by PKC (circles), and both PKC and PKA (squares) in the absence (open symbols) and presence (filled symbols) of $\mathrm{Ca}^{2+}$-calmodulin were determined.

and 1143 of the ruMLCK sequence) that are deleted in psm-, bs- and ruMLCK. While there are notable differences between psm- and cgMLCK near phosphorylation site B (2 polar amino acids substituted with hydrophobic residues and one vice versa), there are no differences in the immediate vicinity of site A.

\section{Discussion}

Myometrial contractility is influenced by fluctuating concentrations of a variety of hormones and agents which alter the intracellular concentrations of cAMP and $\mathrm{Ca}^{2+}$, particularly during gestation and parturition [28]. A mechanism that has been proposed for the modulation of contractile activity of smooth muscles is through the alteration of the activity of MLCK by phosphorylation by protein kinases. In vitro studies have shown that cAMP dependent protein kinase, PKA, and $\mathrm{Ca}^{2+}$ dependent protein kinases, PKC and a multifunctional $\mathrm{Ca}^{2+}$-calmodulin dependent protein kinase, Cam Kinase II, phosphorylate MLCK. PKA modifies two serine residues, sites $\mathrm{A}$ and $\mathrm{B}$ in the absence of $\mathrm{Ca}^{2+}$-calmodulin, but only site $\mathrm{B}$ is phosphorylated when $\mathrm{Ca}^{2+}$-calmodulin is bound to the enzyme [21]. Site A has been localized to the C-terminus of the calmodulin binding site and identified as $S^{815}$ of cgMLCK [29] while site B is $\mathrm{S}^{828}$ [30]. The lack of phosphorylation of site $\mathrm{A}$ in the presence of $\mathrm{Ca}^{2+}$-calmodulin has been attributed to its inaccessibility to the protein kinase when calmodulin is bound to the enzyme. Phosphorylation of site $B$ alone has no effect on its activity while diphosphorylation of MLCK inhibited its activity due to a 10-20-fold decrease of its affinity for $\mathrm{Ca}^{2+}$ and calmodulin [21]. This observation led to the hypothesis that agents which increase the intracel- lular concentration of cAMP modulate contractile activity by altering the MLCK activity through phosphorylation of the kinase. However, there are evidence to suggest that modification of MLCK activity in vivo involves CaM kinase II rather than PKA. Site A is phosphorylated in vitro by Cam Kinase II in the absence of $\mathrm{Ca}^{2+}$-calmodulin with concomitant increase in $\mathrm{K}_{\text {CAM }}$ [31]. The association of site A phosphorylation with the alteration of activity of MLCK is supported by the direct correlation observed by Stull et al. [32] between the MLCK activity and extent of phosphorylation of site A of tracheal MLCK in tissues treated with carbachol, $\mathrm{KCl}$, isoproterenol and PDBu. Furthermore, a decrease in the $\mathrm{K}_{\mathrm{CAM}}$ of MLCK in $\mathrm{KCl}$ stimulated tracheal smooth muscle cells has been attributed to phosphorylation of MLCK by Cam Kinase II [33]. However, the higher activation constant for calmodulin for CaM kinase II (20-100 nM) than for MLCK (1$2 \mathrm{nM}$ ) [33] poses a problem with the suggestion that $\mathrm{CaM}$ kinase II regulates MLCK activity in vivo because calmodulin will bind to MLCK before it activates CaM Kinase II.

Contrary to the stoichiometry of phosphorylation of smooth muscle MLCK by PKA, the extent of phosphorylation of psmMLCK by PKA both in the presence and absence of $\mathrm{Ca}^{2+}$-calmodulin are similar (2.0-2.2 mol phosphate $/ \mathrm{mol}$ MLCK). The sites of phosphorylation of psmMLCK by PKA are most likely to be sites $A$ and $B$ because comparison of the primary sequences of the C-terminal regions of psmMLCK and other MLCK shows high homology and that $S^{815}$ and $S^{828}$ are conserved. Moreover, the tryptic maps of the diphosphorylated psm- and tgMLCK are the same. Thus, the phosphorylation stoichiometry of psmMLCK suggests that binding of $\mathrm{Ca}^{2+}$-calmodulin to psmMLCK does not interfere with the phosphorylation of site $A$ as it does in tgMLCK. The reason for this property is not evident from the primary struc- 


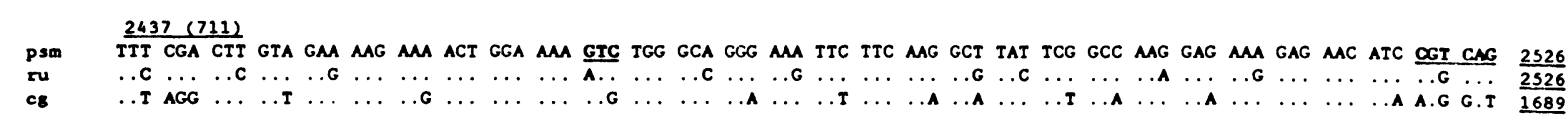

psm GAG ATC AGC ATC ATG AAC TGC CTG CAC CAC CCC AAG CTG GTC CAG TGT GTG GAC GCC TTT GA GAG AAG GCC AMC ATC GTC ATG GTC CTG 2616
TU

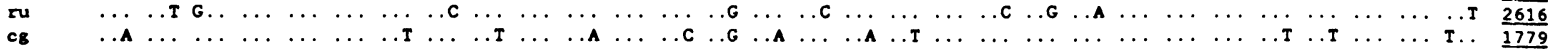

Psm GAG ATT GTG TCT GGG GGC GAG TTG TTC GAG CGC ATC ATT GAT GAG GAC TTC GAG CTG ACG GAG CGG GAG TGC ATC AMG TAC ATG MAG CAG 2706

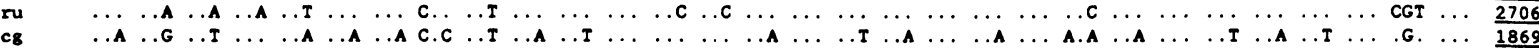

PSm ATC TCG GAG GGG GTG GAG TAC ATC CAC AAG CAG GGC ATC GTC CAC CTG GAC CTC MAG CCC GAG AMC ATC ATG TGT GTC AMC AAG ACA GGC 2796

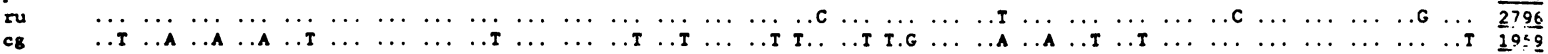

psm ACC $\triangle G G$ ATC AAG CTC ATC GAC TTC GGT CTG GCC AGG AGG TTG GAG MAT GCA GGG TCT CTG AMG GTC CTC TTI GGC ACC CCG GAG TTC GTC 2886

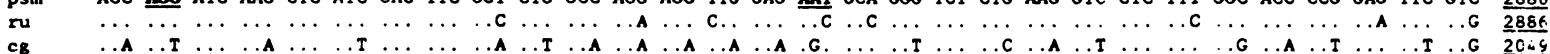

psm GCA CCC GAA GTG ATC AAC TAC GAG CCC ATT GCC TAT GCC ACA GAC ATG TGG AGC ATC GGG GTC ATC TGC TAT ATC CTG GTC AGT GGa CTC 2976

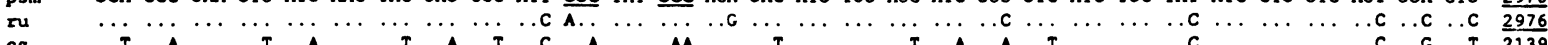

psm TCC CCC TTC ATG GGT GAC AAT GAC AAC GAA ACC ITA GCC AAC GTT ACC TCA GCC ACC TGG GAC TTC GAC SAC GAG GCC TTT GAT GAG ATC 3066

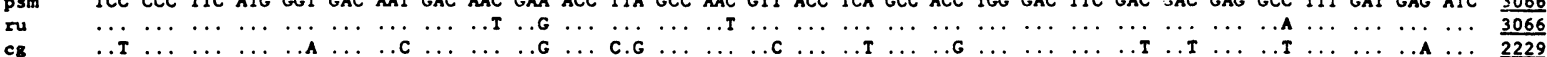

Psm TCT GAT GAC GCC AMG GAT TTT ATC AGC MAC TTG CTG AAG AAG GAT ATT MA MAC CGC TTG MAC TGC ACC CAG TGC CTT CAG CAT CCA TGG 3156

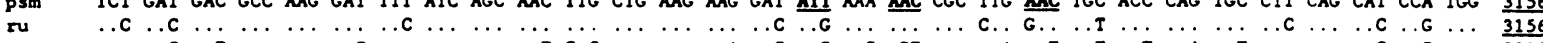

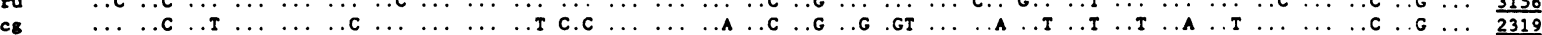

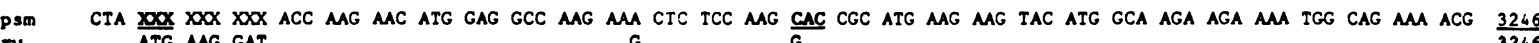

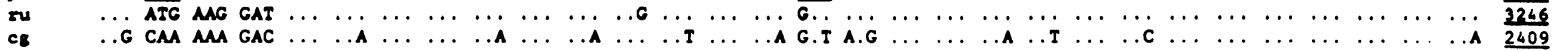

psm GGC MAT GCT GTG AGA GCC ATC GGA AGA CTG TCC TCT ATG GCA ATG ATC TCA GGG CTC AGT GGC AGG AAM TCC TCA ACA GCC TCA CCA ACC 3336

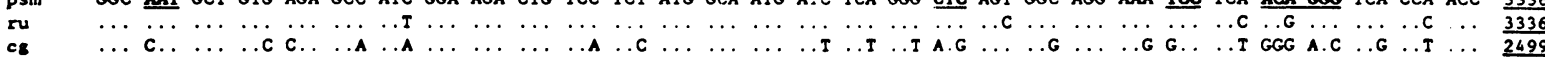

psm AGC CCA GTC MAT GCA GM MA GTA GM ICT GM GM GAT GTC TCC CMA GCT ITC CTG GAG GCT GTT GCT GAG GAG AMG CCC CAT GTA MMA 3426

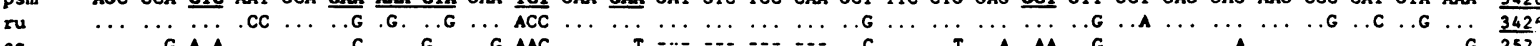

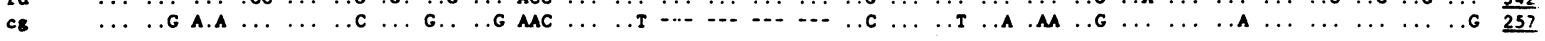

Psm CCC TAC TTT TCC MG ACC ATT OGT GAT TIA GN. GTT GTG GAG GGA AGT GCT GCG AGA TTT GAC TGC MAG ATT GAM GGA TAC CCA GAC CCT 3510

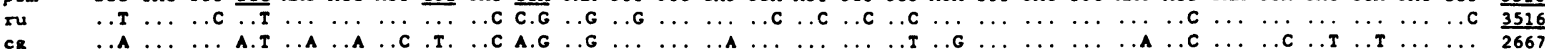

Psm GAN GTC GTC TGG IIC AAA GAT GAC CAM TCA ATC COG GAG TCC CGC CAC ITC CAG ATA GAC TAC CAG GAG GAT GGA MAC TGC TCT TTA ATC 3606

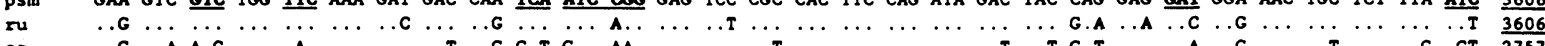

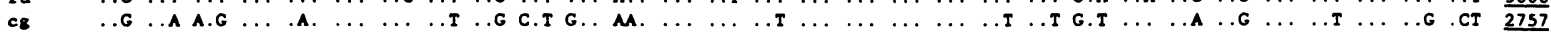

PSm ATT AGT GAT GTT TGC GGG GAT GAT GAT GCC AAG TAC ACC TGC AAG GCT GTC AMC AGT CTC GGA GAN GCC ACC TGC ACA GCA GAG CTC ATC 3696

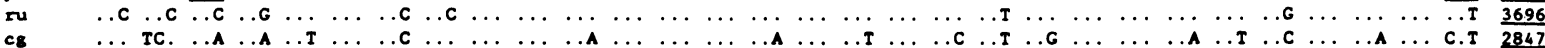

psm GTG GM ACC ATG -..- -..- GAG GM GGA GA GGG GA GGG GGA GAG GAG -.. -.. -.. GAG GAG GMA GAG TGA* 3753

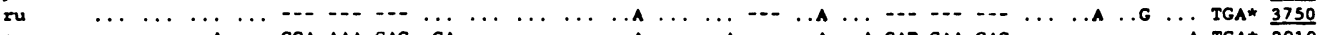

Fig. 7A. Nucleotide sequence of the cDNA encoding the C-terminal region of psmMLCK is aligned with the nucleotide sequences of rabbit uterine smooth muscle (ru) and chicken gizzard (cg) MLCK. All nucleotides are identical (denoted by ·) unless otherwise stated. Letters in bold type and underlined represent triplets in the sheep sequence which encode different amino acids from either the rabbit or chicken sequences. Numbering for rabbit uterine muscle [9] and chicken gizzard [7] sequences are taken from published sources. The numbering of psmMLCK is aligned at bp 2437 of the ruMLCK sequence for comparison purposes. Dashes represent positions where nucleotides need to be added to maintain alignment.

ture of psmMLCK since the sequence of the chicken and sheep MLCK around serine residue $\left(\mathrm{S}^{815}\right)$ are the same. Changes in primary structure near site B include substitution of the hydrophobic amino acids before and after $S^{828}$ with polar amino acids in psmMLCK, a non-conservative substitution of $G^{830}$, and a 4 amino acid insertion between $D^{845}$ and
$\mathrm{A}^{846}$. It is possible that these differences may cause conformational change to make site A accessible to PKA for phosphorylation in the presence of bound calmodulin. It is interesting that two monoclonal antibodies to tgMLCK with epitopes on the tryptic peptide containing the phosphorylatable serine residues did not cross react with psmMLCK. 


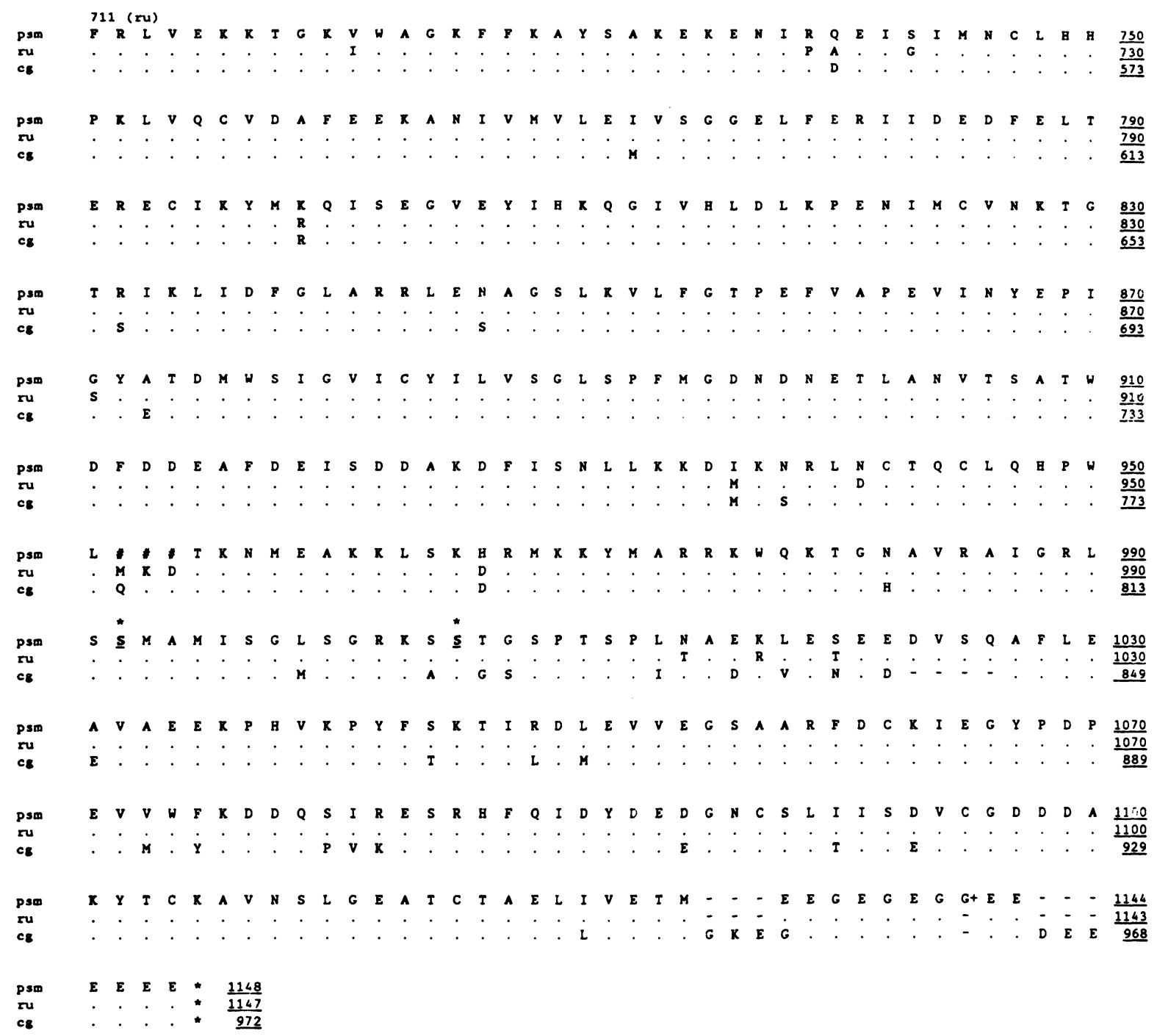

Fig. 7B. Deduced amino acid sequence of the C-terminal region of psmMLCK from the nucleotide sequence of a cDNA encoding this region. The psmMLCK amino acid sequence is aligned to published sequences of rabbit uterine smooth muscle (ru) and chicken gizzard (cg) MLCK. All amino acids are identical unless otherwise stated. Numbering for the ru- and cg MLCK is taken from published sources. The numbering of psmMLCK is aligned at amino acid 711 of the ruMLCK sequence for comparison purposes. Dashes represent positions where the amino acid residues need to be added to maintain alignment. + denotes an additional glycine in psmMLCK not present in chicken or rabbit sequences. The serine residues phosphorylatable by PKA are denoted by asterisks. \#denotes undetermined amino acids.

Although the residues at the calmodulin binding that are essential for binding $\mathrm{L}^{813}, \mathrm{I}^{810}, \mathrm{~W}^{800}, \mathrm{G}^{804}$ and $\mathrm{R}^{812}[35]$, are conserved in the psmMLCK, there are two substitutions in this domain which might affect the binding of calmodulin to the enzyme allowing phosphorylation of site $\mathrm{A}$ in the presence of $\mathrm{Ca}^{2+}$-calmodulin. These amino acids are $\mathrm{D}^{789}$ and $\mathrm{H}^{805}$ which are histidine and asparagine, respectively, in psmMLCK.

Nishikawa et al. [20] have demonstrated that phosphorylation of human platelet MLCK by PKC in the presence of $\mathrm{Ca}^{2+}$-calmodulin incorporated 0.8-1.1 mole phosphate into a site different from those phosphorylated by PKA and did not affect the activity of the enzyme. On the other hand, 2-2.4 moles phosphate were incorporated (sites A and B) in the absence of $\mathrm{Ca}^{2+}$-calmodulin resulting in the inhibition of enzyme activity due to 3.3-fold increase in the concentration of calmodulin required for half maximal activation. Ikebe et al. [26] also observed incorporation of 2 moles phosphate into a mole of tgMLCK in the absence of $\mathrm{Ca}^{2+}$-calmodulin and about 10-fold decrease in the affinity for calmodulin of the diphosphorylated enzyme. But the residues modified by PKC were different from those phosphorylated by PKA. In the presence of $\mathrm{Ca}^{2+}$-calmodulin, no phosphorylation of MLCK was observed. In this study the phosphorylation of psmMLCK by PKC incorporated 2.0-2.1 mole phosphate both in the presence and absence of $\mathrm{Ca}^{2+}$-calmodulin with no effect on the activity of the MLCK suggesting that site A is 
not phosphorylated under these conditions. Indeed, subsequent phosphorylation by PKA incorporated additional phosphate into sites A and B and inhibited the enzyme activity. These results differ from that of Nishikawa et al. [20] and Ikebe $e t$ al. [26] but are consistent with the finding of Stull $e t$ al. [32] that treatment of tracheal muscle with PDBu resulted in phosphorylation of MLCK but no significant phosphorylation was found at site $\mathrm{A}$.

The discrepancy in the extent of phosphorylation of psmMLCK and other smooth muscle MLCK by PKA and PKC may be due to difference in their structures. This is suggested, firstly, by the difference in the molecular weights of avian and psmMLCK. We have previously shown that a 28 $\mathrm{kD}$ peptide is present in the $\mathrm{N}$-terminal region of psmMLCK but not in tgMLCK. It is not obvious how this structure could affect the phosphorylation of residues near the $\mathrm{C}$-terminus of the molecule without knowledge of the three dimensional structure of MLCK. Secondly, some monoclonal antibodies to tgMLCK did not cross-react with psmMLCK. Thirdly, there are notable differences in the primary structures of the C-terminal regions of $\mathrm{cg}$ - and psmMLCK.

Although increase in cAMP levels have been shown to inhibit contraction by decreasing the intracellular $\mathrm{Ca}^{2+}$ concentration $[36,37]$, the possibility that psmMLCK is regulated by PKA phosphorylation cannot be discounted. Our data showing that even in the presence of $\mathrm{Ca}^{2+}$-calmodulin, psmMLCK can be phosphorylated by PKA with subsequent decrease in enzyme activity is consistent with the well known physiologic response of the myometrium to $\beta$-adrenergic agonists. In this smooth muscle, $\beta$-adrenergic agonists produce profound inhibition of both spontaneous contractions and those induced by agents that act by increasing intracellular $\mathrm{Ca}^{2+}$ concentration such as oxytocin, prostaglandin $\mathrm{F}_{2 \alpha}$, and carbachol $[38,39,40]$. Indeed, this ability to relax the myometrium even in the presence of stimulants is used clinically when hypertonus develops during oxytocin administration for labor induction [41].

\section{Acknowledgement}

This work is supported by grants to MDP and SJL from the Medical Research Council of Canada.

\section{References}

1. Hartshorne DJ: Biochemistry of the contractile process in smooth muscle. In: L.R. Johnson (ed.). Gastrointestinal Tract, Second edition, Raven Press, New York 1987, pp 423-482

2. Stull JT, Nunnally MH, Michnoff CH: Calmodulin-dependent protein kinases. In: The Enzymes, Vol. XVII, Academic Press, Inc., Orlando, Florida, 1986, pp 113-166
3. Takio K, Blumenthal DK, Edelman AM, Walsh KA, Krebs EG, Titani $\mathrm{K}$ : Amino acid sequence of an active fragment of rabbit skeletal muscle myosin light chain kinase. Biochemistry 24: 6028-6037, 1985

4. Takio K, Blumenthal DK, Walsh KA, Titani K, Krebs EG: Amino acid sequence of rabbit skeletal muscle myosin light chain kinase. Biochemistry 25: 8049-8057, 1986

5. Edelman AM, Takio K, Blumenthal DK, Hansen RS, Walsh KA, Titani $\mathrm{K}$, Krebs EG: Characterization of the calmodulin binding and catalytic domains in the skeletal muscle myosin light chain kinase. J Biol Chem 260: 11275-11285, 1985

6. Rousch CL, Kennelly PJ, Glaccum MB, Helfman DM, Scott JD, Krebs EG: Isolation of the cDNA encoding rat skeletal muscle myosin light chain kinase. J Biol Chem 263: 10510-10516, 1988

7. Olson NJ, Pearson RB, Needleman DS, Hurwitz MY, Kemp BE, Means AR: Regulatory and structural motifs of chicken gizzard myosin light chain kinase. Proc Natl Acad Sci USA 87: 2284-2288, 1990

8. Shoemaker MD, Lau W, Shattuck RL, Kwiatkowski AP, Matrisian PE, Guerra-Santos L, Wilson E, Lukas TJ, Eldik LJ, Watterson DM: Use of DNA sequence and mutant analyses and antisense oligodeoxynucleotides to examine the molecular basis of nonmuscle myosin light chain kinase autoinhibition, calmodulin recognition, and activity. $\mathrm{J}$ Cell Biol 111: 1107-1125, 1990

9. Gallagher PJ, Herring BP, Griffin SA, Stull JT: Molecular characterization of a mammalian smooth muscle myosin light chain kinase. J Biol Chem 266: 23936-23944, 1991

10. Kobayashi H, Inoue A, Mikawa T, Kuwayama H, Hotta Y, Masaki T, Ebashi S: Isolation of cDNA for bovine stomach $155 \mathrm{kDa}$ protein exhibiting myosin light chain kinase activity. J Biochem 112: 786-791, 1992

11. Pato MD, Lye SJ, Kerc E: Purification and characterization of pregnant sheep myometrium myosin light chain kinase. Arch Bioch Biophy 287: 24-32, 1991

12. Adelstein RS, Klee CB: Purification and characterization of smooth muscle myosin light chain kinase. J Biol Chem 256: 7501-7509, 1981

13. Pato MD, Adelstein RS: Purification and characterization of a multisubunit phosphatase from turkey gizzard smooth muscle. J Biol Chem 258: 7047-7054, 1983

14. Klee CB: Conformational transition accompanying the binding of $\mathrm{Ca}^{2+}$ to the protein activation of $3^{\prime}, 5^{\prime}$-cyclic adenosine monophosphate phosphodiesterase. Biochemistry 16: 1017-1024, 1977

15. Adelstein RS, Conti MA, Hathaway DR, Klee CB: Phosphorylation of smooth muscle myosin light chain kinase by the catalytic subunit of adenosine 3':5'-monophosphate-dependent protein kinase. J Biol Chem 253: 8347-8350, 1978

16. Kinzel V, Kubler D: Single step purification of the catalytic subunit(s) of cyclic 3',5'-adenosine monophosphate-dependent protein kinase(s) from rat muscle. Biochem Biophys Res Comm 71: 257-264, 1976

17. Bradford MM: A rapid and sensitive method for the quantitation of microgram quantities of protein utilizing the principle of proteindye binding. Anal Biochem 72: 248-254, 1976

18. Laemmli UK: Cleavage of structural proteins during the assembly of the head of Bacteriophage T4. Nature (Lond.) 227: 680-685, 1970

19. Witt JJ, Roskoski R Jr: Rapid protein kinase assay using phosphocellulose-paper absorption. Anal Biochem 66: 253-258, 1975

20. Nishikawa M, Shirakawa S, Adelstein RS: Phosphorylation of smooth muscle mosin light chain kinase: comparative study of the phosphorylation sites. J Biol Chem 260: 8978-8983, 1985

21. Conti MA, Adelstein RS: The relationship between calmodulin binding and phosphorylation of smooth muscle myosin kinase by the catalytic subunit of $3^{\prime}: 5^{\prime}$ cAMP-dependent protein kinase. J Biol Chem 256: 3178-3181, 1981

22. de Lanerolle P, Nishikawa M: Regulation of embryonic smooth muscle 
myosin by protein kinase C. J Biol Chem 263: 9071-9074, 1988

23. Chirgwin JM, Pryzbyla AE, MacDonald RJ, Rutter W: Isolation of biologically active ribonucleicx acid from sources rich in ribonuclease. Biochemistry 18: 5294-5299, 1979

24. Sambrook J, Fritsch EF, Maniatis T: In Molecular Cloning, Second edition, Cold Spring Harbor Laboratory Press, New York, 1989

25. Biolojan C, Takai A: Inhibitory effect of marine-sponge toxin, okadaic acid, on protein phosphatases: specificity and kinetics. Biochem J 256: 283-290, 1988

26. Ikebe $M$, Inagaki $M$, Kanamaru K, Hidaka $H$ : Phosphorylation of smooth muscle myosin light chain kinase by $\mathrm{Ca}^{2+}$-activated, phospholipid-dependent protein kinase. J Biol Chem 260: 4547-4550: 1985

27. Araki Y, Ikebe M: Activation of smooth muscle myosin light chain kinase activity by a monoclonal antibody which recognizes the calmodulin-binding region. Biochem J 275: 679-684, 1991

28. Challis JRG, Lye SJ: Parturition. In: J. Clarke (ed.). Reproductive Biology, Oxford University Press, Oxford, 1986, pp 61-129

29. Lukas TJ, Burgess WH, Prendergast FG, Lau W, Watterson DM: Calmodulin binding domains: characterization of a phosphorylation and calmodulin binding site from myosin light chain kinase. Biochemistry 25: 1458-1464, 1986

30. Payne ME, Elzinga M, Adelstein R: Smooth muscle myosin light chain kinase. Amino acid sequence at the site phosphorylated by adenosine cyclic 3',5'-phosphate dependent protein kinase whether or not calmodulin is bound. J Biol Chem 261: 16346-16350, 1986

31. Hashimoto Y, Sordeling TR: phosphorylation of smooth muscle myosin light chain kinase by $\mathrm{Ca}^{2+} / \mathrm{calmodulin}^{2}$ dependent protein kinase-II - comparative study of the phosphorylation sites. Arch Biochem Biophys 278: 41-45, 1990
32. Stull JT, Hsu L-C, Tansey MG, Kamm KE: Myosin light chain kinase phosphorylation in tracheal smooth muscle. J Biol Chem 265: 16683-16690, 1990

33. Tansey MG, Word RA, Hidaka H, Singer HA, Schworer CM, Kamm KE, Stull JT: Phosphorylation of myosin light chain kinase by the multifunctional calmodulin-dependent protein kinase II in smooth muscle cells. J Biol Chem 267: 12511-12516, 1992

34. Bagchi IC, Huang Q, Means AR: Identification of amino acids essential for calmodulin binding and activation of smooth muscle myosin light chain kinase. J Biol Chem 267: 3024-3029, 1992

35. Colbran RJ, Schworer CM, Hashimoto Y, Fong Y-L, Rich DP, Smith MK, Soderling TR: Calcium/calmodulin dependent protein kinase II. Biochem J 258: 313-325, 1989

36. McDaniel NL, Rembold CM, Richard HM, Murphy RA: Cyclic AMP Relaxes Swine Arterial Smooth Muscle Predominantly by Decreasing Cell $\mathrm{Ca}^{2+}$ Concentration. J Physiol 439: 147-160, 1991

37. Tang D-C, Stull JT, Kubota Y, Kamm KE: Regulation of the $\mathrm{Ca}^{2+}$ dependence of smooth muscle contraction. J Biol Chem 267 : 11839-11845, 1992

38. Dokhac L, D'Albis A, Janmot C, Harbon S: Myosin light chain phosphorylation in intact rat uterine smooth muscle: role of calcium and cyclic AMP. J Muscle Res Cell Motility 7: 259-268, 1986

39. Brugger AJ: The mechanism of the utero-inhibitory effect of bsympathomimetics. Pharmac Therap 1: 277-296, 1975

40. Andersson K-E, Bengtsson LPh, Gustafson I, Ingemarsson I: The relaxing effect of terbutaline on the human uterus during term labor. Am J Obstet Gynecol 121: 602-609, 1975

41. Andersson K-E, Ingemarsson I, Persson CGA: Effects of terbutaline on human uterine motility at term. Acta Obsete Gynec Scand 54: 165-172, 1975 